\title{
Late Discovering Spina Ventosa: A Case Report
}

\author{
Sang Nguyen Ngoc (iD) \\ Ha Nguyen Thai' \\ Quang Vu Van (D) \\ Lam Vu Tung' \\ Rang Nguyen $\mathrm{Ngoc}^{2}$ \\ Hung Nguyen $\operatorname{Van}^{3}$ \\ 'Haiphong University of Medicine and \\ Pharmacy, Hai Phong, Vietnam; ${ }^{2}$ An Giang \\ Central General Hospital, An Giang, \\ Vietnam; ${ }^{3}$ National Lung Hospital, Hanoi, \\ Vietnam
}

\begin{abstract}
Introduction: Spina Ventosa is a rare condition that is easy to misdiagnose as other diseases. We present a case of late-diagnosed Spina Ventosa, which had not only the osteoarticular tuberculosis symptoms but also some severe symptoms, including pleural effusion, ascites, and anemia. By intensive treatment, our patient recovered completely.
\end{abstract}

Case Report: A 7-year-old boy was admitted with complaints of painless swelling of metacarpals, metatarsals, and phalanges of his hands and feet and a discharging sinus of the left toe. There was no family or past history of tuberculosis. His immunizations were up to date. General examination revealed that the child had pallor and was emaciated. No lymphadenopathy was detected. Investigations revealed hemoglobin: $74 \mathrm{~g} / \mathrm{l}$, and erythrocyte sedimentation rate (ESR) was $42 \mathrm{~mm} /$ hour. QuantiFERON-TB test was positive. The radiograph showed irregular swelling with sclerosis of the underlying bones. The right-hand $\mathrm{x}$-ray showed cortical destruction, sclerosis, and cystic expansion or right second metacarpal. Chest $\mathrm{x}$-ray indicated pleural effusion. Histopathological examination of specimen from the foot and lung and abdomen fluid confirmed tuberculosis. The child was treated with the first-line tuberculosis treatment regimen (Isoniazid, Rifampicin, Ethambutol, and Pyrazinamide) for two months, followed by Isoniazid, Rifampicin, and Pyrazinamide for a further four months. His lesions disappeared after six weeks of intensive treatment.

Conclusion: A delay in diagnosis and treatment of tuberculosis can lead to systemic manifestations in multiple organs. Despite the delay in diagnosis, this child had a good outcome due to being treated promptly and adequately after the presentation.

Keywords: spina ventosa, osteo-articular tuberculosis, children

\section{Introduction}

Extrapulmonary tuberculosis is less prevalent than pulmonary tuberculosis, with a prevalence of $10 \%$. Spina Ventosa is an uncommon disease caused by tuberculosis bacteria, which causes damage to the bones and skin without a primary site of infection. ${ }^{1,2}$ The long bones are frequently damaged, and there is diffuse sclerosis of the short bones in the hands and feet. Since the development of the epiphyseal centers, Spina Ventosa rarely occurs after the age of 6. It usually manifests itself $1-3$ years after the initial infection. The hematopoietic marrow of tubular bones is favorable for the hematogenous dissemination of tuberculosis to the marrow of this age group. The result is a fusiform swelling of the bone with thinned cortex and a radiolucent marrow space caused by the expansile granulation tissue. Cortical destruction and soft tissue swelling are the inevitable consequences. It generally occurs as a painless swelling of a digit that lasts several months. A mild fever and pain at the affected site are possible. Clinical symptoms such as anorexia and weight loss are also prevalent.

In a medical literature review, there have been several case reports of Spina Ventosa with significant damage to the skeletal system, no cases of severe 
complications in other organs. We reported a boy with Spina Ventosa since the age of one, but it was not diagnosed until he was seven years old when the disease caused severe complications in many organs. After that, the child was intensively treated with anti-tuberculosis drugs and recovered.

\section{Case Report}

A 7-year-old boy presented with swelling of metacarpals, metatarsals, and phalanges of his hands and feet. He had swelling associated with aching pain since he was one year of age. He was diagnosed with polyarthritis at that time. After one month of treatment, his symptoms were not changed, and he was transferred to VNC Hospital. He was diagnosed with Pediatric Skeletal Dysplasia (at that time, the patient had no signs of pleural and peritoneal effusion). There was no improvement after two months of treatment, and he was treated with traditional medicine. A few days before admission to HPC Hospital, all metacarpals, metatarsals, and phalanges of his hands and feet were swollen and painful. Moreover, he had poor appetite, fatigue, and abdominal distension. He was hospitalized in HPC Hospital.

On physical examination, he was thin, and his body weight was $18 \mathrm{~kg}$. The pulse rate was $105 / \mathrm{min}$, and the respiratory rate was $30 / \mathrm{min}$. The body temperature was $37.0^{\circ} \mathrm{C}$. The infection syndromes were unclear. The patient had anemia manifestations: pale skin and mucosa. Physical examination revealed the visibly enlarged distal interphalangeal (DIP) joint of the left middle finger, the proximal interphalangeal (PIP) joint of the right index finger, and the interphalangeal joint of the left great toe with a firm, fusiform swelling that involved the proximal and middle phalanges, with overlying erythema (See Figure 1). The knuckles of his hand and feet were sore and scaly spots on the skin surface. The patient had atrophy of the forearm muscles, arm muscles, thigh muscles, leg muscles on both sides. The joints were moved within normal limits. Tendon reflex was normal. In addition, the patient had splenohepatomegaly, collateral circulation, and ascites. A dull percussion sound was noted in the left lung. History of local trauma, cough, bowel disturbances, and immunosuppression was negative. A provisional diagnosis of polyarthritis was made, and the differential diagnosis was bone cancer.

His complete blood count showed moderate anemia (erythrocyte count $3.71 \times 10^{12} / \mathrm{L}$, hemoglobin of $74 \mathrm{~g} / \mathrm{L}$ ), white blood cells count $7.49 \times 10^{9} / \mathrm{L}$ with $52.7 \%$ neutrophils, and platelets count $410 \times 10^{9} / \mathrm{L}$. His erythrocyte sedimentation rate (ESR) was $42 \mathrm{~mm} /$ hour, and $\mathrm{C}$-reactive protein (CRP) was $24 \mathrm{mg} / \mathrm{L}$. Liver function tests revealed normal bilirubin and transaminases. Serum electrolytes test showed $\mathrm{Na}^{+} 135 \mathrm{mmol} / \mathrm{l} ; \mathrm{K}^{+} 3.4 \mathrm{mmol} / \mathrm{l} ; \mathrm{Cl}^{-} 103 \mathrm{mmol} / \mathrm{l}$; total calcium $2.1 \mathrm{mmol} / 1$; ionized calcium $0.89 \mathrm{mmol} / 1$. The urinalysis test was normal. Abdominal ultrasound showed ascites, hepatomegaly, splenomegaly, and other organs were normal. $\mathrm{X}$-rays of hands and feet showed decreased joint space with periarticular erosions and subluxation, suggesting that the patient might have tuberculous arthritis (See Figure 2). Chest ultrasound revealed left pleural effusion with $3 \mathrm{~cm}$ thick fluid along the middle axillary line, the fifth intercostal space, and revealed a little fluid in the pericardium. Chest X-ray showed pleural effusion (See Figure 3). QuantiFERON-TB test was positive. Rivalta test was positive, much higher in protein content $(46 \mathrm{~g} / \mathrm{l})$

On dermatology examination, there were some skin lesions with fibrinous bases and tuberculous gummas. Because of the clinical characteristics lasting for many years and the laboratory investigations, the patient was diagnosed with Spina Ventosa at a later stage. Eventually, he was transferred to NL Hospital.

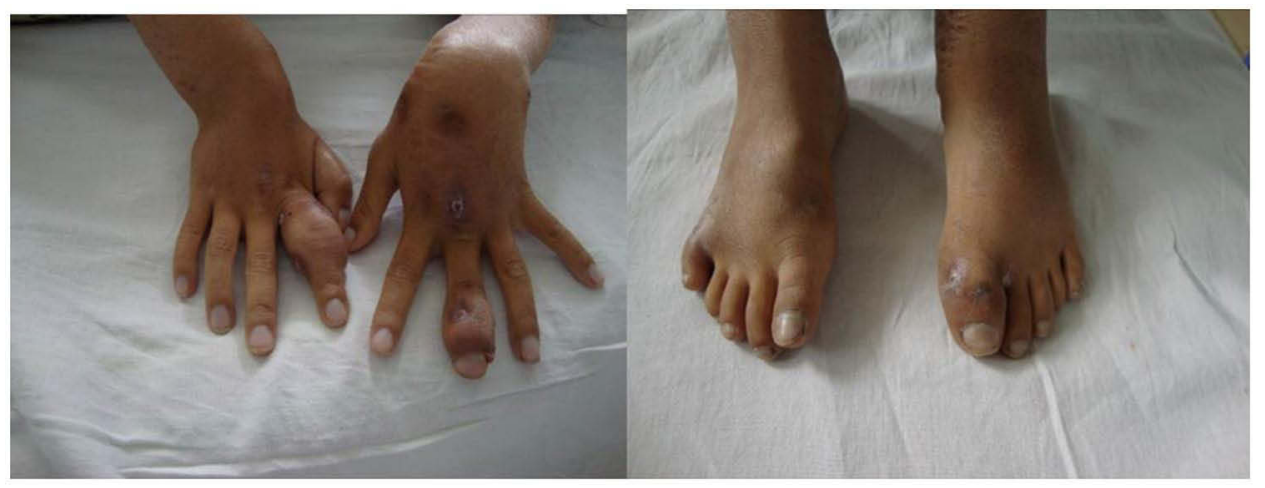

Figure I Two views of hands and feet with swelling of metacarpals, metatarsals, and phalanges. 

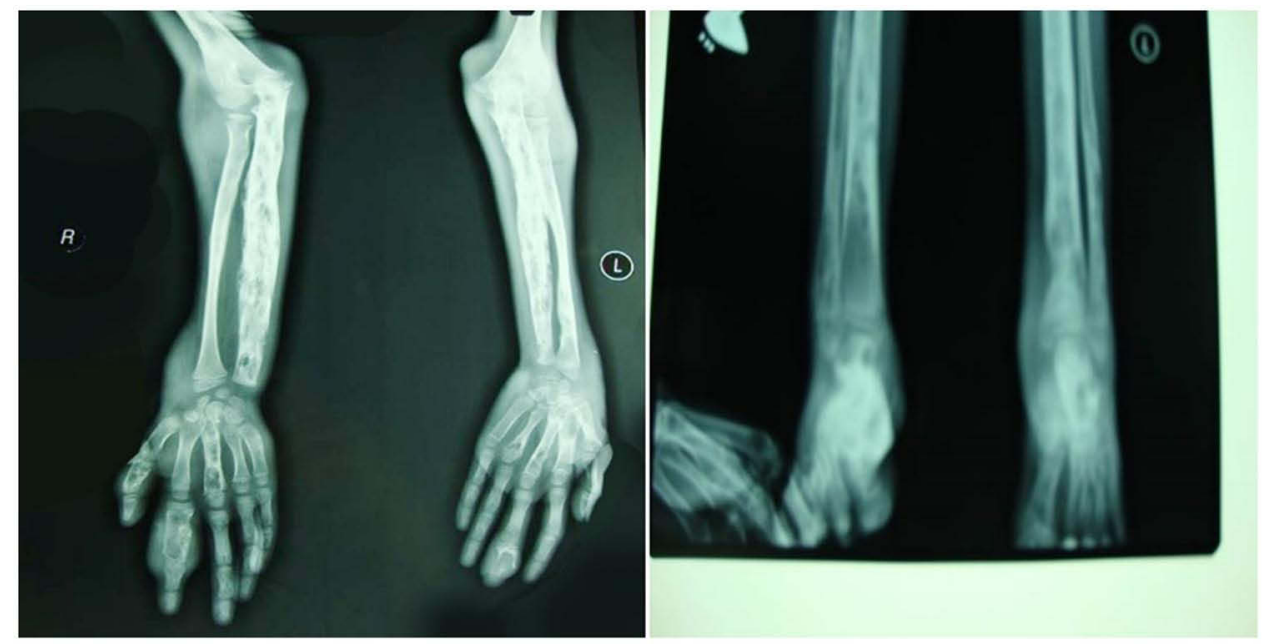

Figure 2 The X-ray images of two upper extremities and lower extremities showed decreased joint space with periarticular erosions and subluxation.

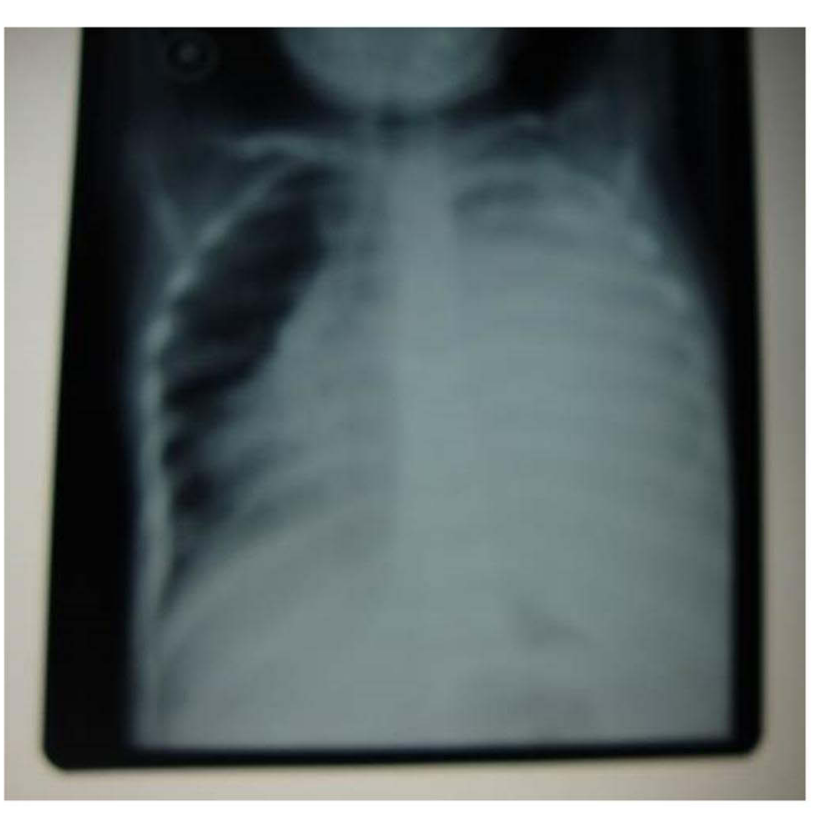

Figure 3 The plain X-ray of the patient's chest showed pleural effusion.

At the NL Hospital, laboratory investigations were as follows: Hands and feet X-rays showed decreased joint space with periarticular erosions and subluxation correlated with the signs of tuberculous arthritis. The chest CT scanner showed pleural effusion. QuantiFERON-TB test was positive $(++)$. The histopathological examination revealed epithelioid granuloma, which indicates tuberculosis. Serum CRP increased to $126 \mathrm{mg} / \mathrm{l}$. Liver and kidney function tests were normal. Abdominal ultrasound showed ascites, hepatomegaly, and splenomegaly.

The child was diagnosed with Spina Ventosa and was treated with anti-tuberculosis drugs - RHZE (Rifampicin,
Isoniazid, Ethambutol, and Pyrazinamide) for two months. His lesions recovered after six weeks of treatment, Ethambutol was removed, and the rest three drugs continued for four months.

After three months of treatment, the patient had fully recovered: no swelling in hands and feet, no pleural effusion, no spleno-hepatomegaly, and no ascites (See Figure 4).

\section{Discussion}

Spina Ventosa occurs in children who have untreated initial pulmonary tuberculosis. ${ }^{3,4}$ In this child, the diagnosis was delayed because the initial infection detection in Spina Ventosa was difficult. In our study, the child was diagnosed after a 6-years of symptoms.

Usually, the skeletal infection becomes symptomatic within 1-3 years after the initial infection. Tubercular dactylitis, or tubercular infection of the metacarpals, metatarsals, and phalanges of the hands and feet, is more frequent in children. ${ }^{5}$ Almost all Spina Ventosa patients have bone and skin manifestations, but no primary infection is found.

During the initial infection, untreated pulmonary tuberculosis spreads to the skeletal system through the lymphohematogenous route. ${ }^{3}$ Spina Ventosa often occurs in $1-5 \%$ of children who have untreated initial pulmonary tuberculosis. ${ }^{4,6}$ Tubercular dactylitis rarely occurs without an initial infection. The majority of initial infection appears in the lung. Hand bones are more commonly affected than foot bones, with the proximal phalanx of the index and the middle finger being the most commonly affected. ${ }^{7}$ 


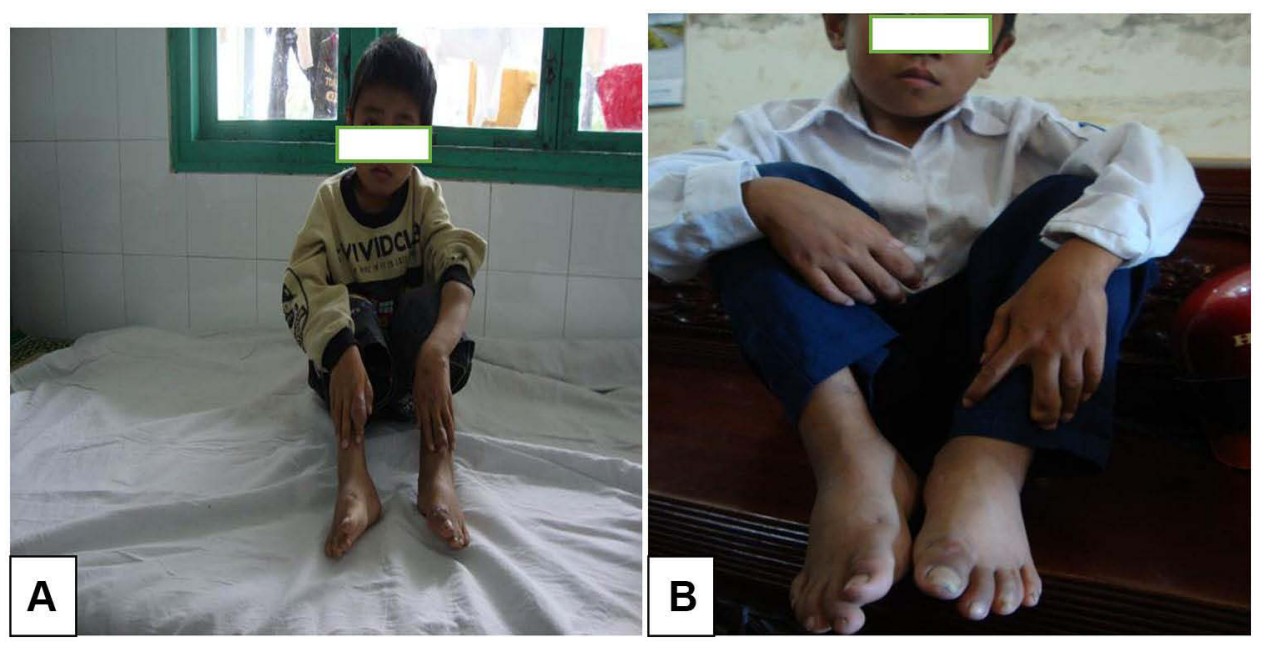

Figure 4 Patient with swelling of both hands and feet before treatment (A) and no swelling in hands and feet, no pleural effusion after successful treatment (B).

In contrast to adults, lesions are located in the peripheral bone among children. ${ }^{8}$ Infections usually occur at the metaphysis of the bone, while diaphyseal lesions are uncommon. ${ }^{9}$ Sclerosis may progress over time in some cases, but it is not a frequent symptom, except during the healing process. ${ }^{10}$ The radiographic feature of the cystic expansion of short tubular bones has led to the name of Spina Ventosa being assigned to tuberculous dactylitis of the short bones of the hand. ${ }^{11,12}$ In this patient, sclerosis and thickening of the affected bones indicated a purulent infection. A perplexing image appears as a result of secondary infection of these infected bones.

Due to its clinical and radiological similarities to other causes of dactylitis, tuberculous dactylitis is often misdiagnosed. Despite the delayed diagnosis of tuberculous dactylitis, the regimen of combined anti-tuberculous drugs would result in a complete cure of the disease. If the patient is appropriately diagnosed and treated, most patients with Spina Ventosa will have a good prognosis. ${ }^{13}$

\section{Conclusion}

Tuberculous dactylitis without initial infection is rare, and delays in diagnosis and treatment, leading to the presence of systemic manifestations in multiple organs. Despite the delay in diagnosis, the child had a good outcome due to being treated properly and promptly upon diagnosis.

\section{Data Sharing Statement}

The data that support the findings of this study are available from the corresponding author upon reasonable request.

\section{Consent for Publication}

Written informed consent was obtained from the patient's parents for publication of this case report and the associated images. A copy of the written consent is available for review by the Editor of this journal. Approval for the study was obtained from Medical Ethics Council of Haiphong University of Medicine and Pharmacy, and informed consent was obtained according to the Declaration of Helsinki. Haiphong Children's Hospital approved all authors to publish the case details.

\section{Disclosure}

The authors declare that they have no competing interests.

\section{References}

1. Bhaskar KT, Bareh J. Tuberculous dactylitis (spina ventosa) with concomitant ipsilateral axillary scrofuloderma in an immunocompetent child: a rare presentation of skeletal tuberculosis. Adv Biomed Res. 2013;2:29. doi:10.4103/2277-9175.107993

2. Sharma S, Sood S, Gupta M. Spina ventosa with lupus vulgaris and lymphadenopathy: multifocal tuberculosis. Indian Dermatol Online J. 2015;6(Suppl 1):S40-S42. doi:10.4103/2229-5178.171042

3. Bandyopadhyay R, Mukherjee A, Mondal RK. Case report: "spina ventosa" tuberculous dactylitis in a 2 year old boy - a very rare disease. Open Orthop J. 2012;6:118-120. doi:10.2174/187432500120 6010118

4. Kushwaha RA, Kant S, Verma SK, Sanjay MS. Isolated metacarpal bone tuberculosis-a case report. Lung India. 2008;25(1):17-19. doi:10.4103/0970-2113.44132

5. Fairag R, Hamdi A. Tuberculous dactylitis: case presentation and functional outcome. $J$ Orthop Case Rep. 2016;6(3):22-24. doi:10.13107/jocr.2250-0685.484

6. Zoga A, Lee VW. Pediatric case of the day. Tuberculosis dactylitis and primary pulmonary tuberculosis. AJR Am J Roentgenol. 1999;173 (3):813, 5-7. doi:10.2214/ajr.173.3.10470936

7. Mohan V, Gupta SK, Agrawal AK. Disseminated multicystic tuberculosis. (A case report). Indian Pediatr. 1980;17(12):987-990. 
8. Salimpour R, Salimpour P. Picture of the month. Tuberculous dactylitis. Arch Pediatr Adolesc Med. 1997;151(8):851-852. doi:10.1001/archpedi.1997.02170450101018

9. Edeiken J, DePalma AF, Moskowitz H, Smythe V. "Cystic" tuberculosis of bone. Clin Orthop Relat Res. 1963;28:163-168.

10. O'Connor BT, Steel WM, Sanders R. Disseminated bone tuberculosis. J Bone Joint Surg Am. 1970;52(3):537-542. doi:10.2106/00004623-197052030-00012

11. Malik S, Joshi S, Tank JS. Cystic bone tuberculosis in children--a case series. Indian J Tuberc. 2009;56(4):220-224.
12. Gyanshankar PM, Dhamgaye TM, Amol BF. Spina ventosa discharging tubercle bacilli--a case report. Indian J Tuberc. 2009;56 (2):100-103.

13. Kothari PR, Shankar G, Gupta A, Jiwane A, Kulkarni B. Disseminated spina ventosa. Indian J Chest Dis Allied Sci. 2004;46 (4):295-296

\section{Publish your work in this journal}

The International Medical Case Reports Journal is an international, peer-reviewed open-access journal publishing original case reports from all medical specialties. Previously unpublished medical posters are also accepted relating to any area of clinical or preclinica science. Submissions should not normally exceed 2,000 words or 4 published pages including figures, diagrams and references. The manuscript management system is completely online and includes a very quick and fair peer-review system, which is all easy to use. Visit http://www.dovepress.com/testimonials.php to read real quotes from published authors. 